\title{
The Wavefunctions and Energy Eigenvalues of the Schrodinger Equation for Different Potentials Due to the Virial Theorem
}

\author{
H. Arslan*, N. Hulaguhanoglu \\ Department of Physics, Bingol University, Bingol, Turkey
}

\begin{abstract}
The derivation of the virial theorem is presented both in classical and quantum mechanical approach. The kinetic energy and potential energy of the mechanical energy is converted to each other due to the virial theorem. Some of the different potentials are considered. For some of these potentials, the wavefunctions and energy eigenvalues of the Schrödinger Equation are derived.
\end{abstract}

DOI: 10.12693 /APhysPolA.127.914

PACS: 03.65. Nk, 03.65.Sq, 03.65.-w, 83.10.Ff

\section{Introduction}

The design of the paper is as follows. In section 2, we wrote the virial theorem both for the classical and quantum mechanical cases by using the works done previously. In section 3, the wavefunctions of the Schrödinger Equation are written for different potentials due to the virial theorem. In section 4 , the energy eigenvalues of the Schrödinger Equation are calculated in the same sense. The conclusion of this work is given in section 5 .

\section{The derivation of the virial theorem}

\subsection{Classical case}

In the references [1-5], the virial theorem is derived as follows:

$$
G=\sum_{i} \boldsymbol{p}_{i} \cdot \boldsymbol{r}_{i}
$$

here $G$ is considered to be a quantity, $\boldsymbol{p}_{i}$ the momentum, and $\boldsymbol{r}_{i}$ the position of the particle in a stable system. Taking the derivative of Eq. 1, we get the equation:

$$
\frac{\mathrm{d} G}{\mathrm{~d} t}=\sum_{i}\left(\frac{\mathrm{d} \boldsymbol{p}_{i}}{\mathrm{~d} t} \cdot \boldsymbol{r}_{i}+\boldsymbol{p}_{i} \cdot \frac{\mathrm{d} \boldsymbol{r}_{i}}{\mathrm{~d} t}\right) .
$$

The second term in the right hand of the Eq. 2 can be written as [5]:

$$
\begin{aligned}
& \sum_{i} \boldsymbol{p}_{i} \cdot \frac{\mathrm{d} \boldsymbol{r}_{i}}{\mathrm{~d} t}=\sum_{i}\left(m \dot{\boldsymbol{r}}_{i}\right) \cdot \dot{\boldsymbol{r}}_{i}=m \dot{r}_{i}^{2}=2 T \text { and } \\
& \sum \dot{\boldsymbol{p}}_{i} \cdot \boldsymbol{r}_{i}=\sum_{i} \boldsymbol{F}_{i} \cdot \boldsymbol{r}_{i} .
\end{aligned}
$$

Here $T$ is the kinetic energy. If the quantity $G$ is bounded in a time interval of $\tau$, one can write:

$$
\frac{1}{\tau} \int_{0}^{\tau} \frac{\mathrm{d} G}{\mathrm{~d} t} d t=\frac{1}{\tau}(G(\tau)-G(0))=0 .
$$

From Eq. 2, it is obvious that

\footnotetext{
* corresponding author; e-mail: hasanars lan46@yahoo.com
}

$$
\frac{1}{\tau} \int_{0}^{\tau} \frac{\mathrm{d} G}{\mathrm{~d} t} d t=2 T+\sum \boldsymbol{F}_{i} \cdot \boldsymbol{r}_{i},
$$

if the momentum is periodic. From Eq. 4 and 5 we get the equation

$$
2 T=-\sum_{i} \boldsymbol{F}_{i} \cdot \boldsymbol{r}_{i}
$$

Equation 6 is the virial theorem derived in the classical case.

\subsection{Quantum mechanical case}

The time dependent Schrödinger Equation is

$$
i \hbar \frac{\mathrm{d} \psi}{\mathrm{d} t}=H \psi \text {. }
$$

The derivative of expectation value of an operator $A$ with respect to time is

$$
i \hbar \frac{\mathrm{d}}{\mathrm{d} t}\langle\psi|A| \psi\rangle=\langle\psi|[H, A]| \psi\rangle .
$$

We let $A=\boldsymbol{r} \cdot \boldsymbol{p}$. Inserting this in Eq. 8, choosing $A$ to be time-independent, and using time-dependent Schrödinger equation we obtain the following equation after some calculations

$$
\langle\psi|[H, A]| \psi\rangle=0 \text {. }
$$

Then the commutation is

$$
\begin{aligned}
& {[H, A]=\left[\frac{p^{2}}{2 m}+V(r), \boldsymbol{r} \cdot \boldsymbol{p}\right]=i \hbar \boldsymbol{r} \cdot \nabla V-\frac{i \hbar}{m} \boldsymbol{p}^{2}=} \\
& i \hbar \boldsymbol{r} \cdot \nabla V-2 i \hbar T=0 .
\end{aligned}
$$

From the last equation, it is obvious that

$$
2\langle T\rangle=\langle\boldsymbol{r} \cdot \nabla V\rangle \text {. }
$$

Equation 10 is the virial theorem derived in the quantum mechanical case.

The quantum mechanical virial theorem, including relativistic form, is widely used in the works for different purposes; for more information see [6-24] and the references therein. 


\section{The wavefunctions of the Schrödinger equation for a potential given by $V(r)=k r^{n}$}

The total mechanical energy of a system is the sum of the kinetic energy $T$ and the potential energy $V$ written as

$$
E=T+V
$$

For a potential

$$
V(r)=k r^{n},
$$

where $k$ is a constant and $n \neq 0$ and an integer, Eq. 11 can be written as

$$
2 T=n V \text {. }
$$

If we take $n=1$ in Eq. 14, we get the total energy of the stable system in terms of potential energy as

$$
E=\frac{3 V}{2} \text {. }
$$

The spatial and time-dependent Schrödinger Equations are

$$
\left[\frac{p^{2}}{2 m}+V(r)\right] \psi=E \psi \text {, and } i \hbar \frac{\mathrm{d} \psi}{\mathrm{d} t}=E \psi,
$$

respectively. By using Eq. 15 in Eq. 16, we can write

$$
\frac{\mathrm{d} \psi}{\mathrm{d} t}=-\frac{3 i}{2 \hbar} V \psi \text {. }
$$

We are now ready to obtain the wavefunction for the potential $V(r)=k r^{n}$ with different $n$ values. For the simplest case, we let the number $n=1$. By putting $V=k r$ in Eq. 17 we obtain the wavefunction as

$$
\psi=\psi_{0} \mathrm{e}^{-i \frac{3 k}{2 \hbar} \int r d t} \text {. }
$$

Defining the constant $k$ as $k^{\prime} \rightarrow \frac{3 k}{2 \hbar}$, the wavefunction is

$$
\psi=\psi_{0} \mathrm{e}^{-i k^{\prime} \int r d t} \text {. }
$$

For different values of the integer $n$, we can obtain different wavefunctions by inserting the defined potentials and integrating Eq. 17. It means that the type of the wavefunction depends on the type of the potential.

\section{The energy eigenvalues of the Schrödinger equation for different potentials}

We can write the Eq. 11 for $n=-1$ as

$$
2 T=-V
$$

the same as derived in the classical case given by the Eq. 6. Then, the mechanical energy of the system due to the virial theorem is

$$
E=T+V=-\frac{p^{2}}{2 m},
$$

where

$$
p \rightarrow-i \hbar \nabla \text {. }
$$

By using Eq. 21 and 22, the Schrödinger Equation becomes

$$
\frac{\hbar^{2}}{2 m} \frac{\mathrm{d}^{2} \psi}{\mathrm{d} r^{2}}=E \psi
$$

If we take $\psi=\mathrm{e}^{k r}$, the energy eigenvalues of the Eq. 23 are

$$
E_{n}=\frac{\hbar^{2} k_{n}^{2}}{2 m} .
$$

Here, it must be noted that the application of the virial theorem to the Schrödinger Equation gives the same eigenvalues as in the quantum mechanics.

Now we rewrite Eq. 11 as

$$
\langle T\rangle=\left\langle\frac{1}{2} r \frac{\mathrm{d} V}{\mathrm{~d} r}\right\rangle \text {. }
$$

Then, the mechanical energy can be written as

$$
E=\left\langle\frac{1}{2} r \frac{\mathrm{d} V}{\mathrm{~d} r}\right\rangle+V .
$$

By using Eq. 26, for a spatial wavefunction $u(r)$, the Schrödinger Equation takes the form

$$
\left[\frac{1}{2} r \frac{\mathrm{d} V}{\mathrm{~d} r}+V\right] u(r)=E u(r) .
$$

Choosing the potential as $V(r)=k r^{n}$ and solving the Eq. 27 for this potential we get the energy eigenvalues

$$
E_{n}=\left(\frac{n+2}{2}\right) k r^{n} \text {. }
$$

If $n=1$, the energy is just in the form of the work done by a force taking the particle to a distance $r$. If $n=-1$, the energy is in the form of the central or attractive potential. Also, the energy eigenvalues are in terms of the potential.

The application of the virial theorem to the Schrödinger Equation is done in the reference [7] in a different way.

We are now looking the energy eigenvalues of the Eq. 27 for a Yukawa potential

$$
V(r)=-\frac{Q}{r} \mathrm{e}^{-k r} \text {. }
$$

By using this potential we get the solution of the Eq. 27 for the energy eigenvalues as

$$
E=\left(\frac{2 k r-1}{2}\right) \frac{Q}{r} \mathrm{e}^{-k r} .
$$

Again, the energy eigenvalues are in terms of the potential.

\section{Conclusions}

The virial theorem is derived in both of the classical and quantum mechanical approaches. Both derivations imply the same validity of the theorem. The theorem is applied to the Schrödinger Equation to obtain the wavefunctions and energy eigenvalues of the equation. As a result of the applications, it is concluded that the wavefunctions are different for each of the chosen potentials and energy eigenvalues are in terms of the potentials.

\section{References}

[1] D. Kuić, Int. J. Theor. Phys. 52, 1221 (2013).

[2] J.D. Stokes, H.P. Dahal, A.V. Balatsky, K.S. Bedell, Phil. Mag. Lett. 93, 672 (2013).

[3] H. Goldstein, Classical Mechanics, AddisonWesley Publishing Company, Inc., Reading (1959). 
[4] S.T. Thornton, J.B. Marion, Classical Dynamics of Particles and Systems, Thomson Learning, Belmont (2004).

[5] H. Arslan, Appl. Math. 4, 688 (2013).

[6] B.Y. Al-Khasawneh, MSc. Thesis, Yarmouk University, Irbid , 6 (2010)

[7] T. Nadareishvili, A. Khelashvili, arXiv preprint arXiv:0907.1824 (2009).

[8] R. Gurtler, D. Hestenes, J. Math. Phys. 16, 573 (2008).

[9] W. Namgung, J. Korean Phys. Soc. 32, 647 (1998).

[10] J.N. Bahcall, Phys. Rev. 124, 923 (1961).

[11] Z. Ru-Zeng,W. Yu-Hua, Q. Jin Chinese Phys. 11, 1193 (2002)

[12] E. Weislinger, G. Olivier, Int. J. Quantum Chem. 8, 389 (1974).

[13] E. Weislinger, G. Olivier, Int. J. Quantum Chem. 9, 425 (1975).
[14] G. Kalman, V. Canuto, B. Datta, Phys. Rev. D $\mathbf{1 3}$ 3493 (1976).

[15] F. Rosicky, F. Mark, J. Phys. B: At. Mol. Phys. 8, 2581 (1975).

[16] A. Barshalom, J. Oreg, High Energy Density Physics 5, 196 (2009).

[17] H. Arslan, J. Mod. Phys. 4, 559 (2013).

[18] H. Arslan, Open Journal of Microphysics 1, 28 (2011).

[19] W. Lucha, Mod. Phys. Lett. A 5, 2473 (1990).

[20] V.M. Shabaev, arXiv preprint arXiv:physics/0211087 (2002).

[21] C. Semay, J. Math. Phys. 34, 1791 (1993).

[22] N.H. March, Phys. Rev. 92, 481 (1953).

[23] M.E. Rose, T.A. Welton, Phys. Rev. 86, 432 (1952).

[24] A.A. Balinsky, W.D. Evans, Lett. Math. Phys. 44 233 (1998). 